\title{
La Belgique francophone accouche douloureusement d'un cours de philosophie et de citoyenneté non désiré par tous
}

\section{Xavier Delgrange}

\section{(2) OpenEdition \\ Journals}

Édition électronique

URL : http://journals.openedition.org/rdr/397

DOI : $10.4000 /$ rdr.397

ISSN : 2534-7462

\section{Éditeur}

Presses universitaires de Strasbourg

\section{Édition imprimée}

Date de publication : 24 avril 2018

Pagination : 107-132

ISBN : 979-10-344-0017-1

ISSN : 2493-8637

\section{Référence électronique}

Xavier Delgrange, «La Belgique francophone accouche douloureusement d'un cours de philosophie et de citoyenneté non désiré par tous », Revue du droit des religions [En ligne], 5 | 2018, mis en ligne le 25 novembre 2019, consulté le 19 novembre 2020. URL : http://journals.openedition.org/rdr/397 ; DOI : https://doi.org/10.4000/rdr.397

\section{(c) (†) (8)}

La revue du droit des religions est mise à disposition selon les termes de la Creative Commons Attribution - Pas d'Utilisation Commerciale 4.0 International - CC BY-NC 4.0. 


\section{LABELGIQUEFRANCOPHONEACCOUCHE DOULOUREUSEMENTD'UN COURSDE PHILOSOPHIEETDECITOYENNETÉ NON DÉSIRÉPARTOUS}

\section{Xavier DELGRANGE}

Université Saint-Louis - Bruxelles et Université libre de Bruxelles

Premier auditeur chef de section au Conseil d'État de Belgique

\section{Résumé}

Empruntant la voie explorée notamment par la Norvège et le Québec, la Belgique francophone met en place depuis 2015, dans l'enseignement officiel obligatoire, un cours de philosophie et de citoyenneté qui se substitue partiellement, pour l'instant du moins, aux cours de religion et de morale. Cette innovation ne concerne pas les établissements libres confessionnels qui privilégient une approche transversale de la philosophie, ce qui soulève la question de l'égalité des élèves. La mise en place du nouveau cours rencontre différentes difficultés, dont la conversion des professeurs de religion et de morale, invités désormais à donner un enseignement neutre.

\section{Abstract}

Following the path explored by Norway and Quebec, French-speaking Belgium has been introducing since 2015 a philosophy and citizenship course in compulsory official education, partially replacing, at least for the time being, courses in religion and morality. This innovation does not concern faithbased private schools that favour a transversal approach to philosophy, which raises the issue of equality of pupils. The implementation of the new course is facing various difficulties, including the conversion of teachers of religion and morality, who are now invited to give neutral instruction. 
$\mathrm{E}$ n Belgique, depuis 1988, l'enseignement n'est plus organisé au niveau fédéral mais relève des trois communautés, flamande, française et germanophone. La réglementation de l'enseignement fait désormais l'objet de décrets (c'est ainsi que l'on désigne les normes législatives adoptées par les communautés ${ }^{1}$ ). Les établissements scolaires primaires et secondaires se divisent en trois réseaux. Les deux premiers sont appelés « officiels» car ils sont organisés par les pouvoirs publics. L'un regroupe les écoles directement organisées par la communauté. L'autre comprend les écoles organisées par les pouvoirs locaux, essentiellement les communes (municipalités). Il est dénommé «officiel subventionné» car il est essentiellement financé par la communauté. Le troisième est dit «libre» car ses écoles ont été créées par des associations privées, essentiellement religieuses catholiques, qui ont donc fait usage de la liberté d'enseignement et de la liberté d'association. Le réseau libre est qualifié de «subventionné » car il est lui aussi largement financé par les communautés. En Communauté française, le réseau libre subventionné scolarise $40 \%$ des élèves du primaire et $60 \%$ des élèves du secondaire, soit globalement la moitié de la population scolaire ${ }^{2}$.

Depuis la rentrée scolaire de 2016, les élèves fréquentant un établissement officiel primaire en Communauté française bénéficient d'un nouvel enseignement: le cours de philosophie et de citoyenneté. Cette réforme a été étendue à l'enseignement secondaire lors de la rentrée de 2017.

L'inscription au programme de l'enseignement obligatoire d'un cours de philosophie, intégrant une analyse objective des religions, était envisagée de longue date ${ }^{3}$. En son principe, elle fut accueillie favorablement par le monde politique. Elle n'a toutefois pas été concrétisée vu son impact budgétaire et la quasi-impossibilité d'alourdir encore la grille scolaire. Pour contourner ces deux obstacles, il fallait non pas ajouter un cours mais le substituer à un

1. La législation de la Communauté française (qui se surnomme Fédération Wallonie-Bruxelles, du nom des deux régions qui la compose) peut être consultée sur le site officiel http:// www.gallilex.cfwb.be [consulté le 3 janv. 2018].

2. Les indicateurs de l'enseignement 2016, p. 74-75: http://www.enseignement.be [consulté le 3 janv. 2018]. Pour plus de précision sur l'organisation de l'enseignement en Belgique, V. X. Delgrange, «La gestion de la diversité à l'école: le "modèle" belge», in L'expression du religieux dans la sphère publique, comparaisons internationales, Paris, La Documentation française, 2016, p. 141-158, spéc. p. 142-145.

3. V. les contributions de J.-L. WOLFS et N. RAES, «Les cours de religion et de morale non confessionnelle et leurs alternatives. Le débat en Belgique francophone: mise en perspective internationale »; C. SÄGESSER, «Vers une adaptation de l'enseignement de la religion et de la morale au paysage convictionnel du $21^{\mathrm{e}}$ siècle»; X. DeLGRANGE, «Les cours de philosophie, les cours philosophiques et les droits de l'homme», in J. LECLERCQ (dir.), Morale et religions à l'école? Changeons de paradigme, Louvain-La-Neuve, Presses universitaires de Louvain, 2015. 
autre enseignement. Les cours de religion et de morale non confessionnelle, improprement appelés cours "philosophiques», se sont vite retrouvés dans le collimateur, d'autant qu'ils sont contestés en leur principe par d'aucuns, comme on va le voir.

Après avoir brièvement évoqué l'organisation des cours «philosophiques » et leur situation actuelle (1), l'on exposera la mise en place de l'enseignement de la philosophie et de la citoyenneté (2) pour enfin envisager les difficultés qui s'annoncent (3).

\section{LES COURS «PHILOSOPHIQUES », UN TABOU DÉSORMAIS CONTESTÉ}

La présence du cours de religion au sein de l'enseignement officiel remonte à l'origine du droit scolaire belge ${ }^{4}$. Il a en effet été introduit par la loi du 23 septembre 1842 sur l'instruction primaire. Il s'agissait d'une loi de compromis entre les deux forces politiques de l'époque, motivée par la volonté d'assurer le «libre choix» des parents afin de respecter leurs convictions philosophiques. Les Libéraux obtenaient le développement d'un enseignement officiel et neutre, s'appuyant sur les communes. En échange, les Catholiques imposaient deux heures de religion, données sous la direction des ministres du culte et sous la surveillance et l'inspection des délégués des chefs de cultes, au sein même de l'école officielle. Il s'agit donc d'un enseignement clairement engagé. Par la suite, vinrent s'adjoindre l'enseignement d'autres religions reconnues ${ }^{5}$ ainsi que de la morale non confessionnelle.

Ce profond enracinement ne suffit toutefois pas à assurer la pérennité des cours philosophiques. Les coups de boutoir viennent de deux côtés.

D'une part, le besoin d'instaurer un cours de philosophie, de citoyenneté et d'histoire des religions dans l'enseignement obligatoire se fait de plus en plus pressant. Chargé de veiller à la concrétisation de la Convention relative aux droits de l'enfant, adoptée par l'ONU le 20 novembre 1989, le Comité des droits de l'enfant insiste sur l'objectif assigné à l'éducation, à savoir la

4. V. X. Delgrange, «La neutralité de l'enseignement en Communauté française », Administration publique (trimestriel), 2007/08, p. 119-160; X. Delgrange et A. Overbeeke, « Le choix entre l'enseignement d'une des religions reconnues et celui de la morale non confessionnelle», in X. Delgrange, L. Detroux et M. El Berhoumi (dir.), Les grands arrêts du droit de l'enseignement, Bruxelles, Larcier, 2017, p. 242-280.

5. Sont actuellement reconnus les cultes catholique, protestant, orthodoxe, anglican, israélite et islamique. 
transmission de valeurs, notamment les droits de l'homme et la citoyenneté ${ }^{6}$. Il exprime dès lors les "préoccupations que lui inspire le fait que l'enseignement des droits de l'homme ne fait pas toujours systématiquement partie des programmes scolaires dans l'ensemble de l'État [belge ${ }^{7} »$. De même, l'Assemblée parlementaire du Conseil de l'Europe invite les États à revoir les cursus scolaires «afin de promouvoir une meilleure connaissance des différentes religions, et que l'éducation religieuse ne se fasse pas au détriment de l'enseignement des religions en tant que partie intégrante de l'histoire, de la culture et de la philosophie de l'humanité ${ }^{8} »$. Ces préoccupations sont relayées en Belgique, notamment par la Commission du dialogue interculturel $^{9}$, version belge de la Commission Stasi, qui s'est tenue en 2005 et qui a été prolongée par les Assises de l'interculturalité ${ }^{10}$ en 2010.

D'autre part, le mouvement laiqque a changé d'attitude par rapport aux cours "philosophiques». Précisons qu'en Belgique, il convient de distinguer les laïcités politique et philosophique. La première - à la française - n'est pas consacrée par la Constitution. Ainsi que le constate le Conseil d'État de Belgique, «la Constitution belge n'a pas érigé l'État belge en un État laïque. Les notions de laïcité, conception philosophique parmi d'autres, et de neutralité sont distinctes ${ }^{11} »$. La laïcité philosophique, constituée en mouvement, se définit comme «l'adhésion à une option philosophique particulière, caractérisée par le refus de toute référence à une vérité révélée ou à l'existence d'entités "surnaturelles" ${ }^{12} »$.

6. NATIONS Unies. COMité deS DROITS DE L'ENFAnt, Observation générale ${ }^{\circ} 1$ (2001), paragraphe 1 de l'article 29: les buts de l'éducation, 17 avr. 2001, CRC/GC/2001/1. V. dans le même sens les recommandations formulées par l'Unesco, not. M. Goucha, La philosophie, une école de la liberté, Paris, UNESCO, 2007; L'enseignement de la philosophie en Europe et en Amérique du Nord, Paris, UNESCO, 2011.

7. Nations Unies. Comité Des DROITS DE L'ENFAnt, «Observations finales: Belgique», 18 juin 2010, CRC/C/BEL/CO/3-4.

8. Conseil de l'Europe. Assemblée parlementaire, Recommandation 1396 du 27 janvier 1999 , «Religion et démocratie». Dans le même sens, la Recommandation 1720 du 4 octobre 2005, «Éducation et religion», la Recommandation 1804 du 29 juin 2007, «État, religion, laïcité et droits de l'homme».

9. Rapport final de la Commission du dialogue interculturel, 2005, p. 91 : http://www.unia. be [consulté le 3 janv. 2018]. V. H. Dumont et X. Delgrange, « Le principe de pluralisme face à la question du voile islamique en Belgique», Droit et Société, n 68, 2008, p. 75-108, spéc. p. 87-95.

10. Rapport final des Assises de l'interculturalité, 2010, p. 40. V. M.-C. Foblet et J.-Ph. SCHREIBER (coord.), Les Assises de l'interculturalité, Bruxelles, Larcier, 2013.

11. CE, 21 déc. 2010, n 210000, § 6.7.2: http://www.raadvst-consetat.be [consulté le 3 janv. 2018].

12. N. Rosa-Rosso et M. Jacquemain, «Les deux laïcités », in M. Jacquemain et N. Rosa-Rosso (dir.), Du bon usage de la laïcité, Bruxelles, Aden, 2008, p. 5 
Dans un premier temps, le mouvement laïque a veillé à ce que la laïcité philosophique soit placée «sur un pied d'égalité » avec les religions ${ }^{13}$, obtenant la révision constitutionnelle de 1993 afin de rémunérer les délégués laiqques au même titre que les ministres des cultes reconnus. Depuis cette révision, la laïcité philosophique est désormais consacrée par la Constitution, son organisation étant reconnue par la loi ${ }^{14}$. Concomitamment, le lien entre le cours de morale et la laïcité organisée était affirmé. En Communauté flamande, à l'instar des cours de religion et dans la ligne de la révision constitutionnelle, le cours de morale est élaboré par un conseil représentant la laïcité organisée, qui intervient également dans la désignation des professeurs de morale. Le cours de morale et les cours de religion sont donc traités identiquement. En Communauté française, la législation ne prévoit pas l'intervention officielle de la laïcité organisée dans l'organisation du cours de morale, mais le conçoit néanmoins comme un cours engagé du côté de la laïcité philosophique ${ }^{15}$. La dénomination constitutionnelle de «cours de morale non confessionnelle» a fait place à celle de « cours de morale inspirée par l'esprit de libre examen », esprit qui a présidé à la création de l'Université libre de Bruxelles ${ }^{16}$. Les programmes de ce cours précisent que celui-ci vise à exercer les enfants «dont les parents se réclament d'une forme de pensée laïque à résoudre leurs problèmes moraux sans se référer à une puissance transcendante ni à un fondement absolu par le moyen d'une méthode de réflexion basée sur le principe du libre examen ${ }^{17}{ }$.

Depuis quelques années, le mouvement laïque prône au contraire la suppression des cours philosophiques, à tout le moins leur renvoi hors de la

13. Développements de la proposition de révision de la Constitution: Doc. parl., Sénat, S.E., 1991-1992, n 100-3/1, p. 3: http://www.senate.be [consulté le 3 janv. 2018].

14. Dans la foulée de la révision de l'article 181 de la Constitution, relatif au financement des cultes, intervenue le 5 mai 1993, une loi du 21 juin 2002 a reconnu le Conseil central laïque, composé du Centre d'action laïque et de l'Unie Vrijzinnige Verenigingen, comme une organisation qui offre une assistance morale selon une conception philosophique non confessionnelle. Ces associations sont communément désignées sous le vocable de «laïcité organisée». Le Centre d'action laïque regroupe 28 associations actives dans les domaines de l'enseignement et de la culture, notamment la Fédération des associations de parents de l'enseignement officiel et la Ligue de l'enseignement: http://www.laicite. be [consulté le 3 janv. 2018].

15. V. X. Delgrange, «Les cours de philosophie, les cours philosophiques et les droits de l'homme», op. cit., p. 106-107.

16. Comp. l'article $24 \S 1^{\mathrm{er}}$ al. 4 de la Constitution et l'article 5 du décret du 31 mars 1994 définissant la neutralité de l'enseignement de la Communauté française.

17. Les programmes de cours peuvent être consultés sur le site de la Communauté française: http://www.wallonie-bruxelles-enseignement.be [consulté le 3 janv. 2018]. 
grille horaire, où ils seraient remplacés par un cours de philosophie et de citoyenneté commun à tous les élèves ${ }^{18}$.

L'on sait qu'aux yeux de la Cour européenne des droits de l'homme, le respect des convictions religieuses et philosophiques des parents, consacré par l'article $2 \mathrm{du}$ premier protocole à la Convention européenne des droits de l'homme, emporte deux exigences. D'une part, dans l'ensemble des enseignements organisés par l'État, l'intégralité des informations et connaissances doivent être « diffusées de manière objective, critique et pluraliste». Au cas où un cours dérogerait à cette exigence, un système de dispense garantissant effectivement le respect des convictions des parents doit être mis en place. D'autre part, l'État doit exercer un contrôle non seulement sur le contenu des programmes mais également sur la manière dont ils sont enseignés dans les classes, afin d'éviter des «abus» qui pourraient heurter les convictions des parents «par imprudence, manque de discernement ou prosélytisme intempestif ${ }^{19}$ ».

Organisant les cours «philosophiques», la Communauté française méconnaissait ces deux exigences strasbourgeoises même si, on va le voir, la première est désormais rencontrée ${ }^{20}$. D'une part, bien qu'officiellement engagés, ces cours ne pouvaient faire l'objet de dispense. D'autre part, ils ne font toujours pas l'objet d'un véritable contrôle émanant de l'autorité publique quant à leur contenu ou à la manière dont ils sont donnés. La loi du Pacte scolaire du 29 mai 1959, qui reste applicable en Communauté française, charge les ministres des cultes ou leurs délégués, nommés sur proposition des chefs de culte, d'enseigner la religion, sous le contrôle des délégués des chefs de cultes, chargés de l'inspection de ces cours ${ }^{21}$. La Communauté française traite

18. V. J. De BRUEKER, secrétaire général du Centre d'action laiqque, «Religions et morale non confessionnelle», in J. LeCLERCQ (dir.), Morale et religions à l'école?, op. cit., p. 91-93. Le Centre d'action laïque a revu ses statuts afin d'abandonner la définition philosophique de la laïcité au profit d'une définition politique (V. H. BARTHOLOMEEUSEN, « La laïcité comme principe universel d'impartialité», Salut E Fraternité, n 98, juill. 2017, p. 4-5: http:// www.calliege.be [consulté le 3 janv. 2018]). Ce faisant, une discordance survient entre la Constitution, qui reconnaît un mouvement représentant la laïcité philosophique et la nouvelle nature du Centre d'action laique.

19. Not. CEDH, Gde ch., 29 juin 2007, $\mathrm{n}^{\circ}$ 15472/02, Folgerø et autres c/ Norvège; CEDH, 9 oct. 2007, $\mathrm{n}^{\circ}$ 1448/04, Hasan et Eylem Zengin c/ Turquie; CEDH, Gde ch., 19 oct. 2012, $\mathrm{n}^{\circ}$ 43370/04, Catan et autres c/ Rép. de Moldova et Russie; CEDH, 16 sept. 2014, n 21163/11, Mansur Yalçın et autres c/ Turquie. V. X. Delgrange et A. Overbeeke, op. cit., p. 262-271.

20. V. X. Delgrange, «Les cours de philosophie, les cours philosophiques et les droits de l'homme», op. cit., p. 98-111.

21. V. X. Delgrange et L. Detroux, «Le statut des professeurs de religion et de morale dans l'enseignement officiel», in X. Delgrange, L. Detroux et M. El Berhoumi (dir.), Les grands arrêts du droit de l'enseignement, op. cit., p. 563-589. À propos du statut actuel des 
pareillement le contenu des cours de religion et de morale, par la non-ingérence, notamment en n'établissant aucun référentiel en cette matière et en n'organisant pas leur contrôle par son inspection. De même, les professeurs de ces cours ne sont pas tenus à la neutralité, mais se voient seulement interdire de dénigrer les positions exprimées dans les « cours parallèles ${ }^{22} »$.

Dès lors, quand lassés par les ambiguïtés de la Communauté française qui, d'un côté, ne tentait plus de cacher le caractère engagé du cours de morale et, de l'autre côté, n'ouvrait aucune possibilité de dispense des cours philosophiques, des parents ont décidé de s'en remettre aux juridictions pour obtenir la fameuse dispense, la laïcité organisée a soutenu ce recours à l'activisme juridictionnel.

Interrogée par le Conseil d'État, la Cour constitutionnelle a, par un arrêt du 12 mars 2015 suivant l'enseignement strasbourgeois, constaté que, dans la logique de la révision constitutionnelle de 1993, la législation de la Communauté française n'assure pas que le cours de morale est donné «de manière objective, critique et pluraliste», si bien que le respect des convictions des parents n'était pas garanti 23 .

\section{LA PHILOSOPHIE ET LA CITOYENNETÉ ENSEIGNÉES À L'ÉCOLE}

L'arrêt de la Cour constitutionnelle a eu un double effet sur le législateur. D'une part, il l'a contraint à réagir dans la précipitation, afin d'éviter la pagaille lors de la rentrée scolaire de septembre 2015. Un mécanisme de dispense des cours «philosophiques» est mis en place, assorti d'un programme d'occupation des élèves dispensés, sous la forme d'un encadrement abordant la philosophie et la citoyenneté (2.1). D'autre part, cet arrêt constituait une opportunité en ce qu'il permettait de débloquer le dossier du cours de philosophie enlisé depuis trop longtemps. Pour ce faire, il fallait profiter du kairos propice à une évolution. Le législateur devait toutefois composer

inspecteurs de religion, V. Cons. const., 27 avril 2017, n 45/2017: http://www.constcourt.be [consulté le 3 janv. 2018].

22. D. 31 mars 1994, art. 5; D. 17 déc. 2003, organisant la neutralité inhérente à l'enseignement officiel subventionné, art. 6. - V. X. Delgrange, « La neutralité de l'enseignement en Communauté française», art. cit., p. 119-160.

23. Cons. const., 12 mars 2015, n 34/2015. V. les commentaires de L.-L. Christians et M. El Berhoumi, «De la neutralité perdue à l'exemption du cours de morale», Journal des Tribunaux, $\mathrm{n}^{\circ} 6606,2015$, p. 437-444; X. Delgrange, «Le sort du cours de morale: activisme juridictionnel contre attentisme politique», Administration publique (trimestriel), 2015/2, p. 241-265: http://www.entre-vues.net [consulté le 3 janv. 2018]. 
avec deux contraintes indépassables: faire de la place dans la grille horaire déjà surchargée des élèves et éviter tout licenciement des professeurs des cours «philosophiques». Dès lors, dans l'enseignement officiel, ces cours sont passés de deux à une heure par semaine (2.2) tandis que leurs titulaires ont été partiellement convertis en professeurs de philosophie et de citoyenneté (2.4). Ces aménagements permettent la création d'un véritable cours dans le réseau officiel, tandis que dans le réseau libre confessionnel, cet enseignement continue à être assuré d'une manière transversale (2.3). Le législateur de 2015 s'est manifestement inspiré des propositions déposées, dès 2004, par le député Richard Miller ${ }^{24}$, philosophe de formation, qui s'appuyait lui-même sur les conclusions du rapport parlementaire déposé en 2000 par la députée Bernadette Wynants ${ }^{25}$.

\subsection{UNE DISPENSE ENCADRÉE DE PHILOSOPHIE ET DE CITOYENNETÉ}

Dès le 14 juillet 2015, la Communauté française adopte un décret organisant, au sein des établissements officiels, une dispense des cours «philosophiques» pour les élèves dont les parents ne se reconnaissent dans aucune des options proposées ${ }^{26}$. Les modalités d'octroi de la dispense satisfont aux conditions énoncées par la Cour européenne des droits de l'homme et rappelées par la Cour constitutionnelle, à savoir que la dispense doit être accordée «sur simple demande, non autrement motivée ${ }^{27}$ ».

24. Proposition de décret introduisant un cours de philosophie et d'histoire culturelle des religions dans le programme du troisième degré de l'enseignement secondaire: Doc. parl., P.C.F., 2003-04, n 488/1; S.E. 2004, n 20/1; 2009-10, n 25/1; proposition de décret modifiant l'article 5 du décret du 31 mars 1994 définissant la neutralité de l'enseignement de la Communauté et l'article 6 du décret du 17 décembre 2003 organisant la neutralité inhérente à l'enseignement officiel subventionné et portant diverses mesures en matière d'enseignement: Doc. parl., P.C.F., 2005-06, n 290/1; 2009-10, n 24/1; proposition de décret visant à instaurer un cours de citoyenneté, de philosophie et d'histoire comparée des religions: Doc. parl., P.C.F., 2014-15, n 71/1.

25. B. Wynants, Rapport introductif portant sur "l'introduction de davantage de philosophie dans l'enseignement, que ce soit à court ou à long terme»: Doc. parl., P.C.F., 2000-2001, $n^{\circ}$ 131/1. V. M. Collin, «De la philosophie à l'école? Contribution au débat» et X. DelGRANGE, "Les cours de philosophie et la Constitution », Cahier du Circ, n 1, 2001: http:// www.circ.usaintlouis.be/la-recherche [consulté le 3 janv. 2018]; La philosophie à l'école, Bruxelles, Luc Pire, 2001.

26. Décret de la Communauté française du 14 juillet 2015 instaurant un mécanisme de dispense pour les cours de religion et de morale non confessionnelle dans l'enseignement organisé par la Communauté française et dans l'enseignement officiel subventionné par la Communauté française.

27. Cons. const., arrêt $n^{\circ} 34 / 2015$, précit. Le formulaire de demande de dispense a été validé par la section du contentieux du Conseil d'État (arrêts n 238344 du 30 mai 2017 et $\mathrm{n}^{\circ} 238400 \mathrm{du} 1^{\text {er }}$ juin 2017). 
La difficulté était d'occuper ces élèves dispensés alors qu'aucun budget n'était disponible pour mettre sur pied une activité ${ }^{28}$. Un «encadrement pédagogique alternatif » est temporairement organisé. Son programme, qui embrasse l'éveil à la citoyenneté et au questionnement philosophique, est aussi ambitieux que son organisation est approximative ${ }^{29}$.

\subsection{FAIRE DE LA PLACE DANS LA GRILLE HORAIRE, LA RÉDUCTION DES COURS «PHILOSOPHIQUES»}

Une question était débattue: le législateur pouvait-il réduire le volume horaire des cours «philosophiques »? En effet, la révision de 1988 a enchâssé dans l'article 24 de la Constitution les éléments essentiels de la loi du Pacte scolaire du 29 mai 1959, qui a mis fin à la seconde guerre scolaire. Or cette loi fixait ce volume à deux heures par semaine. Dès 2010, la section de législation du Conseil d'État, réunie en assemblée générale, avait validé une réduction horaire, pour autant que celle-ci n'ait pas «pour effet que ces cours soient à ce point appauvris» que l'exigence constitutionnelle ne serait pas respectée. L'amputation d'une des deux heures n'a pas un tel effet ${ }^{30}$. Il était donc possible de glaner une heure en faveur d'un cours de philosophie et de citoyenneté. Ce qui fut fait dès la rentrée scolaire 2016 pour le primaire, 2017 pour le secondaire ${ }^{31}$.

Pour les élèves dispensés des cours «philosophiques », l'encadrement pédagogique alternatif est remplacé par une deuxième heure de cours de philosophie et de citoyenneté ${ }^{32}$. L'articulation entre la première heure de cours, suivie par tous les élèves, et la seconde, réservée aux élèves dispensés, n'est toutefois pas précisée. Selon la ministre de l'Éducation de

28. En Communauté flamande, les quelques élèves qui sont dispensés sont livrés à euxmêmes durant les deux heures de cours «philosophiques» (V. C. SÄGESSER, «Les cours de religion et de morale dans l'enseignement obligatoire», Courrier hebdomadaire $d u$ CRISP, 2012/15, p. 22-25; P. LOOBUYCK, «L'enseignement de la religion et de la morale dans la Constitution vu de Flandre: réflexion critique et proposition constructive», in J. LeCLeRCQ (dir.), op. cit., p. 175-191).

29. Art. 8bis $\S 7$ de la loi du Pacte scolaire du 29 mai 1959, tel que remplacé par le décret du 14 juillet 2015.

30. Avis 48.023/AG du 20 avril 2010 sur la proposition du député Miller: Doc. parl., P.C.F., 2009-10, n² 24/2. V. X. Delgrange, «Le sort du cours de morale: activisme juridictionnel contre attentisme politique», art. cit., p. 256-257.

31. Art. 8 al. 3 de la loi du Pacte scolaire, inséré par le décret du 22 octobre 2015 relatif à l'organisation d'un cours et d'une éducation à la philosophie et à la citoyenneté.

32. Art. 8 al. 4 de la loi du Pacte scolaire, inséré par le décret du 22 octobre 2015. 
l'époque, Joëlle Milquet, cette «deuxième heure pourrait être à la première ce que les travaux pratiques sont au cours principal dans l'enseignement supérieur ${ }^{33} »$.

\subsection{UN COURS DANS L'OFFICIEL ET UNE ÉDUCATION DANS LE LIBRE}

L'organisation de l'enseignement obligatoire est assurée par le décret du 24 juillet 1997 définissant les missions prioritaires de l'enseignement. Ce décret prévoit l'établissement de référentiels qui définissent les compétences que les élèves doivent acquérir au cours de leurs études, sur la base desquels les réseaux établissent les programmes de cours. L'élaboration de ces référentiels est confiée à des groupes de travail composés de représentants des réseaux, de l'administration, de l'inspection ainsi que de la société civile ${ }^{34}$.

L'on sait qu'un important débat divise les pédagogues quant à la manière d'enseigner la philosophie et la citoyenneté à l'école. Pour les uns, ces matières doivent faire l'objet d'une éducation abordée de manière transversale dans différents cours du programme. Pour les autres, il faut leur consacrer un cours spécifique $^{35}$. La Communauté française a longtemps privilégié l'approche transversale ${ }^{36}$. Actuellement encore, le Secrétariat général de l'enseignement catholique (SeGEC), qui fédère le réseau des écoles libres catholiques, est opposé à la création d'un cours spécifique de philosophie et de citoyenneté, estimant que ces matières sont mieux enseignées de manière transversale dans l'ensemble des disciplines, spécialement le cours de religion mais également

33. Rapport de la Commission de l'enseignement: Doc. parl., P.C.F., 2015-16, n 171/3, p. 12.

34. V. X. Delgrange, "Analyse juridique de l'élaboration du décret "missions" ", in H. Dumont et M. Collin (dir.), Le décret du 24 juillet 1997 définissant les missions prioritaires de l'enseignement. Approche interdisciplinaire, Bruxelles, Publ. Saint-Louis, 1999, p. 19-78, spéc. p. 71-75. M. El BeRHOUMI, Le régime juridique de la liberté d'enseignement à l'épreuve des politiques scolaires, Bruxelles, Bruylant, 2013, p. 602-606.

35. À propos de la philosophie, V. le rapport Wynants, précit. À propos de la citoyenneté, V. le rapport Eurydice, L'éducation à la citoyenneté en Europe, 2012, p. 18-24: http:// eacea.ec.europa.eu/education/eurydice/documents/thematic_reports/139FR.pdf [consulté le 3 janv. 2018]. En France, depuis le Rapport DeBray de 2002 (L'enseignement du fait religieux dans l'école laïque, Paris, La Documentation française), l'approche transversale est privilégiée (V. not. J.-P. Willaime, «L'enseignement relatif aux religions en Europe: évolutions et enjeux», Administration É Éducation, 2015/4, Laïcité, école et religions, p. 141-147).

36. Outre le décret «missions», V. le décret du 12 janvier 2007 relatif au renforcement de l'éducation à la citoyenneté responsable et active au sein des établissements organisés ou subventionnés par la Communauté française, où une approche interdisciplinaire est privilégiée: «les compétences relevant de la thématique de la citoyenneté relèvent de plusieurs, voire de l'ensemble des champs disciplinaires » (Exposé des motifs: Doc. parl., P.C.F., 2006-07, $\mathrm{n}^{\circ} 321 / 1$, p. 5). 
ceux de français, histoire, éducation artistique ${ }^{37} \ldots$ Il a obtenu que, dans la première mouture du projet, l'instauration d'un cours de philosophie et de citoyenneté ne devait concerner que l'enseignement officiel. Les élèves de l'enseignement libre bénéficieraient d'une éducation à la philosophie et à la citoyenneté, non pas sous la forme d'un cours mais déclinée dans les différents cours et activités, sur la base d'un cadre général interréseaux établi par des groupes de travail composés de représentants des réseaux, des religions et du cours de morale ${ }^{38}$...

La section de législation du Conseil d'État a néanmoins estimé que réserver un cours spécifique aux seuls élèves des établissements officiels emportait une discrimination des élèves des établissements libres qui en étaient privés ${ }^{39}$. Il faut en effet concilier la liberté active d'enseignement, garantie par l'article $24 \& 1^{\text {er }}$ al. $1^{\text {er }}$ de la Constitution, qui «assure le droit de créer - et donc de choisir - des écoles fondées sur une philosophie confessionnelle ou non confessionnelle déterminée ${ }^{40} »$, d'une part, avec le droit fondamental de l'enfant à l'enseignement, consacré par l'article $24 \S 3$ de la Constitution, et le libre choix des parents, garanti par l'article $24 \S 1^{\text {er }}$ al. 2 de la Constitution, qui requiert que ceux-ci puissent librement choisir l'école que fréquenteront leurs enfants, sans autre considération que leurs convictions ${ }^{41}$.

Or l'article $24 \& 3$ de la Constitution requiert que le droit à l'enseignement s'exerce «dans le respect des libertés et droits fondamentaux», ce qui implique notamment la prise en compte des principes inscrits à l'article $29 \S 1^{\text {er }}$ de la Convention relative aux droits de l'enfant, qui assigne notamment comme objectifs à l'éducation, d'«inculquer à l'enfant le respect des droits de l'homme et des libertés fondamentales», "le respect de ses parents, de son identité, de sa langue et de ses valeurs culturels», et de le préparer «à assumer les responsabilités de la vie dans une société libre,

37. E. Michel, «Citoyenneté et tradition chrétienne de l'éducation», Entrées libres, $\mathrm{n}^{\circ} 111$, 2016, p. 3; M.-N. LOVENFOSSE, «EPC. L'enseignement catholique n'est pas en reste!», Entrées libres, $\mathrm{n}^{\circ} 111,2016$, p. 4-5; C. VAN DE WERVE, «Philosophie et citoyenneté. L'affaire de tous!», Entrées libres, n 120, 2017, p. 6-7: http://www.entrees-libres.be [consulté le 3 janv. 2018].

38. Le projet inscrivait ce cadre général dans le décret du 12 janvier 2007.

39. Avis 57.989/2 du 7 septembre 2015 sur un avant-projet devenu le décret de la Communauté française du 22 octobre 2015 relatif à l'organisation d'un cours et d'une éducation à la philosophie et à la citoyenneté: Doc. parl., P.C.F., 2015-16, n 171/1, p. 24-32.

40. Cons. const., 8 mai 2014, $n^{\circ} 80 / 2014$, B.10.1.

41. V. X. Delgrange et M. El Berhoumi, «Les droits en matière d'enseignement", in M. Verdussen et N. Bonbled, Les droits constitutionnels en Belgique. Les enseignements jurisprudentiels de la Cour constitutionnelle, du Conseil d'État et de la Cour de cassation, Bruxelles, Bruylant, 2011, p. 1175-1294, spéc. p. 1211-1219. 
dans un esprit de compréhension, de paix, de tolérance, d'égalité entre les sexes et d'amitié entre tous les peuples et groupes ethniques, nationaux et religieux». L'article $29 \S 2$ de la Convention relative aux droits de l'enfant garantit la «liberté des personnes physiques ou morales de créer et de diriger des établissements d'enseignement», mais à la condition que «les principes énoncés au $\S 1^{\text {er }}$ soient respectés ${ }^{42}$.

Pour déterminer si la distinction établie entre les élèves selon qu'ils fréquentent un établissement officiel ou libre est justifiable, le Conseil d'État commence par identifier les objectifs poursuivis par le Parlement et entérinés par le Gouvernement de la Communauté française:

«L'objectif principal de l'instauration d'un cours de philosophie et de citoyenneté est de "favoriser les conditions permettant aux élèves provenant d'horizons différents de construire ensemble une société pluraliste, durable et harmonieuse".

Il est en effet souhaitable d'offrir aux élèves un espace commun dans lequel ils pourront découvrir les différents courants philosophiques et religieux, ainsi que les textes fondateurs des sociétés démocratiques. Ils pourront y débattre en toute liberté, développer leur sens critique et argumentaire, et apprendre à découvrir comment divergences et convergences peuvent s'articuler.

Le repli sur soi peut susciter comportements violents, incivisme, intolérance, et difficultés à se remettre en question. Ce nouveau cours devra permettre aux élèves de multiplier les points de vue et de se forger une opinion dont la force proviendra de ce qu'elle procède d'un cheminement personnel (écoute de l'autre, connaissance, débat, expérience) ${ }^{43}$.»

Encore que le Gouvernement n'explicite pas pourquoi il faut abandonner l'option transversale au profit d'un cours spécifique ${ }^{44}$, le Conseil d'État constate que le projet ne permet pas d'atteindre ces objectifs d'une manière équivalente dans les différents réseaux puisque seuls les élèves de l'enseignement officiel bénéficieront d'un cours spécifique, faisant l'objet d'une évaluation permettant de s'assurer qu'ils ont acquis les savoirs et compétences établis par les référentiels. Il estime que cette différence ne peut être justifiée

42. Un raisonnement similaire peut être tenu au départ de l'article $13 \S 1^{\text {er }}$ du Pacte international relatif aux droits économiques, sociaux et culturels. V. L.-L. Christians et $X$. Delgrange, «Le contrôle du respect des droits fondamentaux dans les enseignements dispensés », in X. Delgrange, L. Detroux et M. El Berhoumi (dir.), Les grands arrêts du droit de l'enseignement, op. cit., p. 284-301.

43. Rapport sur les travaux du groupe de travail relatif à l'introduction d'un cours de philosophie et citoyenneté: Doc. parl., P.C.F., 2014-2015, n 150/1.

44. Le rapport Wynants et les propositions Miller à l'origine du projet gouvernemental l'exposent toutefois, estimant notamment que l'approche transversale requiert des moyens plus conséquents et impose des procédures plus lourdes. 
par le souci de respecter la liberté d'enseignement dont jouissent les établissements libres ou le libre choix des parents. Ces libertés doivent en effet s'incliner devant l'intérêt supérieur de l'enfant, consacré par la Convention relative aux droits de l'enfant ${ }^{45}$.

Le législateur a refusé de suivre le Conseil d'État. Il n'a pas imposé la création du cours de philosophie et de citoyenneté au réseau libre - ni même permis aux établissements qui le souhaiteraient -, considérant que ce réseau pouvait - et même devait - maintenir les deux heures de la grille horaire dévolues au cours de religion, ce qui ne lui permettait pas d'intégrer le nouveau cours ${ }^{46}$.

Afin de néanmoins prendre en compte les objections du Conseil d'État, le législateur a sensiblement renforcé le cadre législatif de l'éducation à la philosophie et à la citoyenneté dans les établissements libres.

Cette éducation est rendue obligatoire pour tous les établissements, qui se voient imposer non plus un simple cadre général, mais un véritable « référentiel unique interréseaux garantissant que les mêmes objectifs d'éducation, de compétences et de savoirs seront atteints quel que soit le réseau ou l'établissement». Cette éducation a un contenu très ambitieux, décliné selon deux axes, l'éducation philosophique et éthique (connaissance des différents courants de pensée, philosophies et religions, discernement éthique, respect du pluralisme, gestion des conflits, participation à des débats) et l'éducation au fonctionnement démocratique (vivre ensemble, citoyenneté, connaissance de la démocratie, des institutions, des droits de l'homme ${ }^{47}$ ).

Les référentiels interréseaux sont établis par des groupes de travail ad hoc, dont la composition vise à «assurer le pluralisme et une représentation tant des réseaux que des universités ${ }^{48}{ }$, puisqu'ils sont notamment composés de

45. Après avoir rappelé qu'aux yeux de la Cour européenne des droits de l'homme, «lorsqu'au lieu de le conforter, les droits des parents entrent en conflit avec le droit de l'enfant à l'instruction, les intérêts de l'enfant priment», la Cour constitutionnelle estime que «la liberté active de l'enseignement, conçue comme la liberté de dispenser un enseignement selon ses conceptions idéologiques, philosophiques et religieuses n'est pas absolue; elle doit en effet se concilier avec le droit à l'enseignement des enfants et avec l'objectif d'ouvrir l'esprit des enfants au pluralisme et à la tolérance, qui sont essentiels à la démocratie» (Cons. const., 21 mai 2015, n 60/2015, B.14.2. et B.17.5.1. V. L.-L. Christians et X. Delgrange, art. cit., p. 298-301).

46. Exposé des motifs du projet devenu le décret de la Communauté française du 22 octobre 2015: Doc. parl., P.C.F., 2015-16, n 171/1, p. 6.

47. Art. 60bis \& 3 du décret «missions».

48. Déclaration de la ministre Milquet, Rapport de la Commission de l'éducation (Doc. parl., P.C.F., 2015-16, n 171/3), p. 13. 
professeurs de philosophie des différentes universités ${ }^{49}$. Ils sont approuvés par décret ${ }^{50}$.

Dans les établissements libres confessionnels, l'inspection de l'éducation à la philosophie et à la citoyenneté sera assurée conjointement par l'inspecteur de cette éducation et par l'inspecteur du cours qui l'accueille, essentiellement l'inspecteur de religion ${ }^{51}$.

Dans tous les réseaux, l'éducation à la philosophie et à la citoyenneté fait partie de la formation obligatoire et est soumise à évaluation. Dans les établissements libres confessionnels, l'évaluation est assurée par les titulaires des cours qui accueillent cette éducation, principalement les professeurs de religion ${ }^{52}$. Néanmoins, la philosophie ne fait pas partie des matières faisant l'objet de l'évaluation externe qui ponctue le parcours des élèves, notamment en fin de primaire et de secondaire ${ }^{53}$.

Afin d'organiser le cours de philosophie et de citoyenneté, les établissements officiels bénéficient d'un financement spécifique, tant pour l'encadrement de ce cours que pour assurer l'adaptation des programmes et le soutien pédagogique. Le Conseil d'État s'est interrogé à deux reprises sur la validité de ce régime spécifique au regard du principe d'égalité qui doit valoir entre les établissements scolaires, par-delà les réseaux ${ }^{54}$. Le SeGEC a saisi la Cour constitutionnelle de cette question ${ }^{55}$.

49. V. l'audition des responsables des groupes de travail: Doc. parl., P.C.F., 2015-16, n² 262/1.

50. V. deux décrets du 19 juillet 2017, le premier «portant confirmation de l'arrêté du Gouvernement de la Communauté française du 22 mars 2017 déterminant le référentiel des socles de compétences en éducation à la philosophie et à la citoyenneté et prévoyant une procédure de dérogation au référentiel», le second "portant confirmation de l'arrêté du Gouvernement de la Communauté française du 19 avril 2017 déterminant le référentiel des compétences terminales en éducation à la philosophie et à la citoyenneté et prévoyant une procédure de dérogation au référentiel».

51. Art. 4 du décret du 22 octobre 2015. Les modalités de cette inspection conjointe devaient être fixées par un arrêté du Gouvernement de la Communauté française, adopté au plus tard le $1^{\text {er }}$ septembre 2016. V. la circulaire $n^{\circ} 6118$ du 22 mars 2017, «Visites des expertsinspecteurs chargés de construire un projet d'inspection de l'éducation à la philosophie et citoyenneté dans l'enseignement primaire et secondaire de tous les réseaux » : http:// www.gallilex.cfwb.be [consulté le 3 janv. 2018].

52. Art. 60 bis $\S 1^{\text {er }}$ du décret «missions». V. l'exposé des motifs du projet devenu le décret du 22 octobre 2015: Doc. parl., P.C.F., 2015-16, n 171/1, p. 7.

53. V. le décret du 2 juin 2006 relatif à l'évaluation externe des acquis des élèves de l'enseignement obligatoire et au certificat d'études de base au terme de l'enseignement primaire.

54. Avis 59.405/2 du 13 juin 2016 sur un avant-projet devenu le décret du 13 juillet 2016 relatif à la mise en ouvre d'un cours de philosophie et de citoyenneté dans l'enseignement fondamental ainsi qu'au maintien de l'encadrement pédagogique alternatif dans l'enseignement secondaire: Doc. parl., P.C.F., 2015-16, n 312/1; avis 61.576/2 du 21 juin 2017 sur un avant-projet devenu le décret du 19 juillet 2017 relatif à la mise en œuvre d'un cours 


\subsection{LA CONVERSION DES PROFESSEURS DES COURS «PHILOSOPHIQUES»}

Dès l'abord ${ }^{56}$, il fut garanti aux professeurs des cours «philosophiques » que la réduction du volume horaire de leur cours n'aurait aucune incidence sur leur emploi. Ils sont recrutés en priorité pour donner le nouveau cours de philosophie et de citoyenneté, si nécessaire au détriment d'autres enseignants mieux à même d'assurer cet enseignement, par exemple des philosophes qui n'auraient pas été recrutés pour donner les cours «philosophiques ${ }^{57}$ ».

Les professeurs de religion estiment que leurs homologues de morale sont favorisés car il faut être titulaire d'un diplôme de niveau baccalauréat pour pouvoir enseigner la philosophie et la citoyenneté ${ }^{58}$. Or une grande majorité des professeurs de religion ne détiennent qu'un diplôme délivré par les autorités de culte, qui ne permet pas de satisfaire à cette condition. Ils ont introduit un recours devant la Cour constitutionnelle pour faire annuler cette exigence ${ }^{59}$.

Contraint d'agir dans la précipitation, le législateur a dû mettre la charrue avant les bœufs. Les universités ainsi que les «hautes écoles»-qui forment les enseignants du primaire et de la première partie du secondaire - œuvrent à la mise en place d'un certificat en didactique du cours de philosophie et citoyenneté, qui ne sera exigé des enseignants qu'à la rentrée $2021^{60}$. Les professeurs de religion et de morale de l'enseignement officiel bénéficieront d'un crédit d'heures pour suivre cette formation.

de philosophie et de citoyenneté dans l'enseignement secondaire et portant diverses adaptations dans l'enseignement fondamental: Doc. parl., P.C.F., 2016-17, n 494/1, p. 117 et s.

55. Req. $\mathrm{n}^{\circ} 6547,25$ nov. 2016. V. P. Boulllon, «Le Segec dénonce un traitement inégal entre réseaux», Le Soir, 6 déc. 2016.

56. Déjà dans «Fédérer pour réussir», la Déclaration de politique communautaire 2014-2019 (le contrat de gouvernement): Doc. parl., P.C.F., S.E. 2014, p. 10: «En aucun cas, cette réforme ne pourra entraîner la perte d'emploi pour les enseignants concernés en place.»

57. D. 13 juill. 2016 précit. V. l'exposé des motifs: Doc. parl., P.C.F., 2015-16, n 312/1, p. 18 et s. V. S. ARSĖNE, «La rentrée du désarroi et de la colère», La Libre Belgique, 12 sept. 2017.

58. Titre II du décret du 13 juillet 2016 relatif à la mise en œuvre d'un cours de philosophie et de citoyenneté dans l'enseignement fondamental ainsi qu'au maintien de l'encadrement pédagogique alternatif dans l'enseignement secondaire; chapitre II du décret du 19 juillet 2017 relatif à la mise en œuvre d'un cours de philosophie et de citoyenneté dans l'enseignement secondaire et portant diverses adaptations dans l'enseignement fondamental.

59. Req. $\mathrm{n}^{\circ} 6550,25$ nov. 2016. V. B. D'OTREPPE, «Le cours de citoyenneté discrimine-t-il les profs de religion?», La Libre Belgique, 28 nov. 2016.

60. Art. 24bis du décret du 11 avril 2014 réglementant les titres et fonctions dans l'enseignement fondamental et secondaire organisé et subventionné par la Communauté française, inséré par le décret du 13 juillet 2016. 
L'option d'attribuer le nouveau cours prioritairement aux titulaires des cours «philosophiques» rend très malaisée la garantie de la neutralité de ce cours, exigence qui est pourtant à l'origine de sa création. L'on a vu que ces titulaires sont recrutés en vue de dispenser un cours engagé. Comment dès lors les convertir en enseignants neutres?

Le Conseil d'État avait déjà attiré l'attention du Parlement sur cette difficulté à l'occasion de l'analyse de la proposition «Miller ${ }^{61}$. Il a rappelé cette préoccupation lors de l'examen du décret du 22 octobre 2015:

«Confier ce nouveau cours aux actuels maîtres et professeurs de religion ou de morale non confessionnelle ne pourrait être acceptable que moyennant des modalités permettant de s'assurer que le cours de philosophie et de citoyenneté sera dispensé, dans l'enseignement officiel, "conformément à l'obligation de neutralité en vigueur dans l'enseignement public, notamment en prévoyant que les enseignants concernés soient, pour ces cours, soumis en principe au statut applicable à leurs collègues titulaires des autres enseignements obligatoires" ${ }^{62}$.»

Si l'adaptation des décrets "neutralité» et des statuts ne soulevait guère de difficultés, il fallait encore gérer le fait que ces enseignants ne seraient convertis à la neutralité que dans le cadre du nouveau cours de philosophie et de citoyenneté, mais qu'ils recouvreraient leur caractère engagé lorsqu'ils retrouveraient leur enseignement de la religion ou de la morale.

Le législateur a imposé des contraintes particulièrement lourdes aux enseignants du primaire. Un régime d'incompatibilité a été introduit, qui interdit à un enseignant de donner un cours de religion ou de morale et un cours de philosophie et de citoyenneté dans la même implantation scolaire ${ }^{63}$. Si cette règle permet de sauvegarder quelque peu l'apparence de neutralité de l'enseignant - pour autant que les élèves ignorent que leur instituteur enseigne dans une autre école ou implantation -, elle ne le préserve pas des risques de schizophrénie, d'autant que par leur objet, le cours neutre et le cours engagé se rejoignent largement. Le Conseil d'État a considéré que cette mesure, «en ce qu'elle tend à tenir compte de contingences pratiques liées aux conditions de travail et qu'elle se situe dans un contexte particulier de transition », paraissait admissible au regard des exigences de la neutralité de

61. Avis 48.023/AG précit. V. X. Delgrange, «Les cours de philosophie, les cours philosophiques et les droits de l'homme», op. cit., p. 112-117.

62. Avis 57.989/2 précit., qui cite l'avis 48.023/AG.

63. Art. 23, 24, 29 et 34 du décret de la Communauté française du 13 juillet 2016. V. l'exposé des motifs du projet: Doc. parl., P.C.F., 2015-16, n 312/1, p. 6-7. 
l'enseignement ${ }^{64}$. Elle complique toutefois lourdement la tâche des enseignants concernés, qui doivent multiplier les lieux de travail, certains devant exercer dans quinze écoles différentes, parfois très distantes. Un collectif de professeurs de morale et de religion a dès lors introduit un recours devant la Cour constitutionnelle contre cette incompatibilité ${ }^{65}$.

Manifestement instruit de cet écueil, mettant en ouvre le cours de philosophie et de citoyenneté dans l'enseignement secondaire, le législateur a largement assoupli l'incompatibilité puisqu'à ce niveau elle se résume à l'interdiction pour un enseignant de donner à la fois le cours de morale ou de religion et le nouveau cours à un même élève. Partant du constat que «ni la Constitution, ni les décrets relatifs à la neutralité dans l'enseignement officiel ne font de distinction, en ce qui concerne l'exigence de neutralité des enseignants, entre l'enseignement primaire et l'enseignement secondaire », le Conseil d'État a invité le législateur à justifier cette différence de traitement entre les enseignants du primaire et du secondaire ${ }^{66}$. L'exposé des motifs, néanmoins, "justifie la déclinaison différente du principe d'incompatibilité entre le fondamental et le secondaire par l'âge de l'élève. En effet, on estime que l'élève de l'enseignement secondaire a plus de capacité et de discernement et peut donc mieux faire la distinction entre son professeur», selon que celui-ci enseigne la philosophie de manière neutre ou la religion/morale de manière engagée ${ }^{67}$. Il est vrai que, dans la logique de la Convention relative aux droits de l'enfant ${ }^{68}$, les décrets «neutralité» invitent à prendre en compte

64. Avis 59.405/2, précit., p. 62.

65. Req. $n^{\circ}$ 6613, 10 févr. 2017. Leur avocat, Jean Bourtembourg, dénonce un compromis politique qui génère une discrimination: «soit les profs de morale et de religion sont tellement contaminés par leur matière qu'ils sont incapables de donner le cours de citoyenneté. C'est vexatoire mais ça aurait une logique dans la bêtise. Soit ils peuvent donner cours de citoyenneté. Mais alors le fait qu'ils le donnent dans la même classe ou une classe différente n'a rien à voir.» (S. MERGEN et s. FACINELLI, "Cours de citoyenneté: "un décret injuste et discriminatoire" ", RTBF info, 8 févr. 2017).

66. Avis 61.576/2 du 21 juin 2017, précit., p. 122-123.

67. Exposé des motifs du projet devenu le décret du 19 juillet 2017 : Doc. parl., P.C.F., 2016-17, n 494/1, p. 12.

68. Le Comité des droits de l'enfant insiste sur le fait que l'intérêt supérieur de l'enfant doit s'apprécier compte tenu de «l'âge et [du] degré de maturité» de celui-ci (Observation générale $n^{\circ} 14$ (2013) sur le droit de l'enfant à ce que son intérêt supérieur soit une considération primordiale, CRC/C/GC/14). Devant apprécier le caractère prosélyte du port du voile par une institutrice, la Cour européenne a également pris en compte l'âge des enfants: "la requérante a enseigné dans une classe d'enfants entre quatre et huit ans et donc d'élèves se trouvant dans un âge où ils se posent beaucoup de questions tout en étant plus facilement influençables que d'autres élèves se trouvant dans un âge plus avancé. » (CEDH, déc. 15 févr. 2001, n 42393/98, Dahlab c/ Suisse). V. encore J. Woenrling, «La 
l'évolution de la personnalité des élèves. Il est vrai également que la difficulté s'estompera à mesure que la règle qui veut qu'un professeur qui opte pour le nouveau cours le fasse pour toute sa charge ${ }^{69}$, sera effectivement appliquée.

\section{LES ZONES D'OMBRE SUBSISTANTES, AUTANT D'ÉCUEILS?}

Outre le contentieux actuellement pendant devant la Cour constitutionnelle évoqué plus haut, l'organisation de l'éducation à la philosophie et à la citoyenneté laisse subsister certaines zones d'ombre qui pourraient constituer autant d'écueils susceptibles de remettre en cause la validité de l'édifice devant la Cour constitutionnelle, voire d'amplifier les critiques à l'égard des cours "philosophiques». Le principe d'égalité, qu'il se décline à l'égard des élèves, des enseignants ou des établissements scolaires, est-il strictement respecté (3.1) ? La neutralité du cours de philosophie et de citoyenneté est-elle suffisamment garantie (3.2) ? À propos des cours «philosophiques», qu'en est-il de leur contrôle et quel est désormais le statut du cours de morale (3.3)?

\subsection{LE PRINCIPE D'ÉGALITÉ EST-IL RESPECTÉ À L'ÉGARD DE TOUS SES DESTINATAIRES?}

La question de l'égalité entre établissements scolaires, d'un côté, entre professeurs, de l'autre, est déjà pendante devant la Cour constitutionnelle. Subsiste la différence de traitement entre les élèves selon qu'ils fréquentent l'enseignement officiel, où ils bénéficient d'un cours spécifique de philosophie et de citoyenneté, ou l'enseignement libre confessionnel, où cette matière fait l'objet d'une éducation transversale. L'égalité des élèves par-delà les réseaux est-elle suffisamment garantie par le cadre législatif imposé au réseau libre dans la mise en œuvre de cette éducation?

Selon la ministre de l'Éducation de l'époque, Joëlle Milquet, «la différence, objective, qui subsiste provient de l'impossibilité constitutionnelle de toucher aux deux heures de religion dans l'enseignement libre confessionnel cumulée à l'impossibilité financière d'ajouter une heure de cours dans la grille horaire de tous les élèves ${ }^{70} »$. Selon l'exposé des motifs du décret du 22 octobre 2015 :

religion dans les décisions relatives à la santé et à l'éducation des enfants en droit canadien et québécois», Revue du droit des religions, n 3, mai 2017, p. 173-174.

69. D. 19 juill. 2017, précit.

70. Rapport de la Commission de l'enseignement: Doc. parl., P.C.F., 2015-16, n 171/3, p. 14. 
«La différence objective liée à l'engagement confessionnel présent dans l'enseignement libre confessionnel par rapport aux autres types d'enseignement et à l'existence spécifique qui lui est liée de l'organisation de deux heures de religion justifie le maintien d'un traitement différencié uniquement dans la mise en œuvre du référentiel commun. Ce traitement différencié reste par ailleurs marginal et le principe de proportionnalité est également respecté car il ne s'agit que de l'équivalent d'une heure par semaine ${ }^{71} »$.

L'affirmation selon laquelle la Constitution s'opposerait à ce que le volume horaire du cours de religion dans l'enseignement libre confessionnel soit réduit à une heure n'est en rien étayée. Une chose est la volonté des établissements confessionnels d'inscrire deux heures de religion à leur grille horaire. Autre chose est l'obligation pesant sur la Communauté française de financer ces deux heures. L'on n'aperçoit pas en quoi la Constitution imposerait un traitement différent selon les réseaux, ni pourquoi elle permettrait au législateur de déterminer le volume d'enseignement qu'il subventionne, sauf en matière de religion ${ }^{72}$.

Les justifications citées plus haut ne sont donc guère convaincantes ${ }^{73}$. D'autres pourraient toutefois permettre aux distinctions d'éviter la qualification de discriminations. L'on pourrait ainsi, au nom de la liberté d'enseignement, considérer que les approches séparée et transversale de l'éducation à la philosophie et à la citoyenneté présentent des intérêts pédagogiques équivalents pour autant qu'elles soient pilotées par des référentiels suffisamment convergents. Certes, il demeure étrange de voir, dans l'enseignement confessionnel, l'objectif de «connaissance, dans une perspective historique et sociologique, des différents courants de pensée, philosophies et religions ${ }^{74}$ », assuré dans le cadre du cours de religion catholique, islamique, ou autre, suivant la confession de l'établissement. Il ne faut toutefois pas perdre de vue que, dans les établissements confessionnels, ce sont l'ensemble des enseignements et des enseignants qui sont engagés religieusement ${ }^{75}$. En principe, un professeur de

71. Ibid., p. 8 .

72. V. X. Delgrange et M. El Berhoumi, «Les droits en matière d'enseignement », art. cit., p. 1232-1233; M. El Berhoumi et L. VANCRAYEBeCK, Droit de l'enseignement en Communauté française, Bruxelles, Bruylant, 2015, p. 185-189.

73. V. en ce sens les justifications d'un amendement tendant à étendre le cours de philosophie et citoyenneté à l'ensemble des établissements subventionnés: Rapport de la Commission de l'Éducation: Doc. parl., P.C.F., 2015-16, n 171/3, p. 15-16.

74. Art. 60bis $\& 31^{\circ}$ a) du décret «missions».

75. Selon le projet éducatif de l'enseignement catholique, la mission de l'école chrétienne est l'évangélisation: «L'école chrétienne a mission d'annoncer la Bonne Nouvelle du Christ. 
français ou d'histoire n'est pas moins engagé qu'un professeur de religion. Il est d'ailleurs fréquent, dans ce réseau, que le premier enseigne également la religion. Contester que l'histoire et la sociologie des religions puissent être enseignées dans le cadre du cours de religion reviendrait à remettre en cause la légitimité d'un enseignement confessionnel. Or la liberté d'enseignement, qui comprend celle d'ouvrir des établissements confessionnels, est garantie par la Constitution belge ${ }^{76}$.

À tout le moins, il conviendra de veiller à ce que le contrôle prévu par les décrets sur la manière dont le réseau libre permet à ses élèves d'atteindre les objectifs en matière d'éducation à la philosophie et à la citoyenneté soit effectivement mis en place. L'on songe notamment à l'intervention de l'inspection qui, pour l'heure, semble demeurer dans les limbes.

Reste à voir si ces questions pourraient devenir contentieuses, ce qui supposerait que quelqu'un justifie de l'intérêt requis pour porter cette distinction devant la Cour constitutionnelle.

Une autre distinction qui, à ce jour, n'a guère attiré l'attention, est celle qui est introduite entre les élèves dispensés et ceux qui suivent un cours de religion ou de morale. Les premiers bénéficieront de deux heures pour acquérir leurs compétences en matière de philosophie et de citoyenneté tandis que les seconds devront se contenter d'une heure. Comment cette différence sera-t-elle prise en compte au moment de l'évaluation? Les élèves suivant les cours "philosophiques» ne voient-ils pas leur barque plus chargée que les dispensés, puisqu'ils doivent en outre satisfaire à l'évaluation du cours de religion ou de morale?

À cette fin, elle entretient vivante la mémoire de l'événement fondateur, toujours actuel: la vie, la passion, la résurrection de Jésus-Christ [...]. L'école chrétienne trouve ainsi sa référence essentielle dans la personne de Jésus et dans les signes qu'en a gardés la tradition vivante de l'Église. Elle a donc la conviction qu'elle n'éduque pleinement qu'en évangélisant. » (Mission de l'école chrétienne. Projet éducatif de l'enseignement catholique, nov. 2014, p. 3: http://www.enseignement.catholique.be [consulté le 3 janv. 2018]).

76. C'est ainsi que la Cour suprême du Canada, dans son arrêt du 19 mars 2015, a donné raison au collège Loyola qui entendait être dispensé de donner le cours d'éthique et de culture religieuse neutre pour maintenir le cours de religion catholique engagé (V. S. BERNATChEZ et M.-P. ROBERT, «Canada: l'école peut enseigner la religion, mais le conseil municipal ne peut prier», Revue du droit des religions, n 2, nov. 2016, p. 185-187). 


\subsection{LA NEUTRALITÉ ET LA FORMATION DES TITULAIRES DU COURS DE PHILOSOPHIE ET DE CITOYENNETÉ EST-ELLE SUFFISAMMENT GARANTIE?}

Il eut évidemment été plus simple d'assurer la cohérence de la mise en place du cours de philosophie et de citoyenneté s'il n'avait pas fallu agir dans l'urgence ni veiller à préserver l'emploi des titulaires des cours philosophiques. L'on aurait alors pu mettre les bœufs devant la charrette et commencer par construire une formation à destination des futurs enseignants, tout comme on aurait pu estimer qu'une formation en philosophie suffisait pour enseigner... la philosophie.

$\mathrm{Si}$, comme on l'a vu, le sort inconfortable des enseignants du primaire est entre les mains de la Cour constitutionnelle, la très légère incompatibilité imposée aux enseignants du secondaire n'a pas encore été contestée. Le seraitelle, quelle serait l'analyse de la Cour constitutionnelle? Cela dépendrait notamment de son appréciation de l'incompatibilité pesant sur l'enseignement primaire.

\subsection{QUEL AVENIR POUR LES COURS « PHILOSOPHIQUES»?}

La Communauté française commence à se doter des armes lui permettant de contrôler effectivement les cours "philosophiques » afin de s'assurer qu'ils sont donnés dans le respect des droits fondamentaux des élèves. Elle a ainsi revu le statut des professeurs de religion afin, d'un côté, de restreindre le pouvoir des autorités de culte à leur égard et, d'autre part, de renforcer les exigences en termes de titres pédagogiques ${ }^{77}$. Cette réforme ne s'appliquera toutefois pleinement que lorsque les inspecteurs des cours «philosophiques » seront intégrés dans le service général de l'inspection, ce qui contribuerait encore plus grandement à un contrôle effectif ${ }^{78}$. Cette réforme n'est toutefois pas annoncée.

En outre, rien ne se profile s'agissant d'un contrôle des référentiels et des programmes des cours de religion et de morale. Les professeurs des

77. Décret du 30 juin 2016 rendant applicable aux maîtres et professeurs de religion le décret du 11 avril 2014 règlementant les titres et fonctions dans l'enseignement fondamental et secondaire organisé et subventionné par la Communauté française et portant diverses mesures en matière de titres et fonctions. Le Conseil d'État a estimé que ce décret va dans le sens d'une meilleure protection des droits des élèves et d'une amélioration de la qualité de l'enseignement: avis 59.026/2 du 13 avril 2016: Doc. Parl., P.C.F., 2015-2016, n 301/1, p. 63. V. X. Delgrange et L. Detroux, art. cit., p. 563-589.

78. V. en ce sens l'avis 59.026/2, précit. 
cours «philosophiques» eux-mêmes demandent à la Communauté française qu'elle établisse pareils référentiels et programmes, en collaboration avec les autorités de cultes ${ }^{79}$. Faudra-t-il attendre une nouvelle poussée d'activisme juridictionnel pour y parvenir? Ou est-ce le signe de la volonté de ne pas maintenir ces cours?

Le statut actuel du cours de morale devrait fasciner le théoricien du droit. Voici en effet une autorité, la Communauté française, par définition neutre, chargée par la Constitution d'organiser un enseignement neutre, qui conçoit, sans intervention extérieure officielle, un cours non neutre mais engagé du côté de la laïcité philosophique. Les mystères de la génétique ou de la transsubstantiation...

$\mathrm{Au}$ vu des lacunes que présentent toujours les cours «philosophiques» en termes de respect des droits fondamentaux des élèves, malgré l'instauration d'une dispense, il était prévisible que le débat relatif à la suppression des cours "philosophiques» et leur remplacement total par un cours «objectif, critique et pluraliste», pour reprendre le vocabulaire strasbourgeois, qui aborderait la philosophie, la citoyenneté et l'histoire des religions ${ }^{80}$, reviendrait rapidement à l'agenda. Le Mouvement réformateur (parti libéral) a déposé, le 7 décembre 2017, une proposition de décret faisant passer le volume horaire du cours de philosophie et de citoyenneté à deux heures par semaine tout en maintenant un cours "philosophique» d'une heure, mais qui deviendrait facultatif ${ }^{81}$.

La suppression des cours «philosophiques » présenterait l'avantage considérable de mettre fin à la ségrégation et donc à l'étiquetage des élèves en fonction des convictions affichées par leurs parents. En effet, actuellement, dans les écoles officielles, les élèves sont séparés pour suivre les cours "philosophiques», où l'on aborde pourtant les questions relatives au «vivre

79. V. X. Delgrange, "Les cours de philosophie, les cours philosophiques et les droits de l'homme», art. cit., p. 102-104.

80. Encore que cet aspect est ignoré des objectifs assignés au cours de philosophie et de citoyenneté.

81. Proposition de décret modifiant diverses dispositions relatives aux cours de philosophie et citoyenneté, de religion et de morale non confessionnelle: Doc. parl., P.C.F., 2017-2018, $n^{\circ} 568 / 1$. Si les partis écologiques et socialistes, notamment, se sont montrés ouverts à la discussion et si le Centre d'action laïque a salué l'initiative tout en regrettant qu'elle ne soit pas étendue au réseau libre («Le MR propose de rendre optionnels les cours de religion dans l'enseignement officiel », Le Vif, 8 déc. 2017: http://www.levif.be [consulté le 3 janv. 2018]), la constitutionnalité de cette proposition a été contestée par le porteparole des évêques de Belgique (M. VAN LiER, «Quel avenir pour les cours de religion?», CathoBel, 11 déc. 2017: http://www.cathobel.be [consulté le 3 janv. 2018]). 
ensemble ${ }^{82}$ ». Les tenants du maintien de ces cours avancent qu'ils constituent un lieu où les élèves en recherche d'identité peuvent se construire, dans leur dimension spirituelle, auprès d'un professeur empathique, apte à les guider vers la modération et la tolérance. Ainsi, le professeur de religion islamique ou protestante serait plus convaincant que celui de biologie, froidement neutre, pour détourner certains élèves attirés par le radicalisme du créationnisme $^{83}$. Pour l'année 2017-2018, en Communauté française, seuls $10 \%$ des élèves ont demandé à être dispensé de ce cours ${ }^{84}$.

\section{POUR CONCLURE}

\section{On l'a déjà relevé,}

«en Belgique, même si la loi est formellement un acte unilatéral, elle est plus souvent présentée comme le fruit d'un accord contractuel entre des groupements. Au lieu de partir de l'État pour descendre dans la société, la culture politique s'y construit à partir du bas, de la pluralité des traditions religieuses, idéologiques et régionales, pour remonter vers le politique et l'État. En effet, même si les sources supérieures du droit public, en Belgique comme ailleurs, sont toutes marquées du sceau de l'unilatéralité, la réalité sociopolitique du système belge de la décision publique est caractérisée de manière massive par la logique de la négociation et du compromis ${ }^{85} »$.

82. Au Québec, au début de ce siècle, le cours de religion engagé a fait place à un programme d'éthique et de culture religieuse neutre, essentiellement afin de promouvoir l'égalité des élèves et favoriser le «vivre-ensemble» (S. TREMBLAY, «Pluralisme, droits fondamentaux et citoyenneté: les termes d'une nouvelle orientation pour l'enseignement de la religion au Québec », Éducation et sociétés, n²7, 2011/1, p. 161-176). Ce nouveau cours est toutefois contesté à la fois par ceux qui y voient une atteinte à la liberté religieuse comme par ceux qui, estimant que la religion n'a pas sa place à l'école, dénoncent un retour déguisé à la confessionnalité scolaire, tandis que d'autres encore dénoncent un endoctrinement à l'idéologie du multiculturalisme (M. Estivalèzes, S. Tremblay et M. Milot, « Le cours d'Éthique et culture religieuse: un révélateur des tensions entourant la place de la religion à l'école? », Centre d'études ethniques des universités montréalaises, nov. 2013: http:// www.ceetum.umontreal.ca. [consulté le 3 janv. 2018]).

83. V. in J. LeClerCQ (dir.), op. cit., les contributions de H. Dumont, « Cours de religion ou de morale non confessionnelle: les limites de l'alternative constitutionnelle et les pistes pour en sortir», p. 132-135; G. RAINOTTE, «Un point de vue protestant», p. 217-219.

84. C. SäGESSER, «La fréquentation des cours de religion et de morale dans l'enseignement francophone en Belgique», Observatoire des religions et de la laïcité, 22 déc. 2017: http:// www.o-re-la.org [consulté le 3 janv. 2018].

85. H. Dumont et X. Delgrange, «Le principe de pluralisme face à la question du voile islamique en Belgique», Droit É Société, nº 68, 2008, p. 82-83. 
L'élaboration de la législation de la Communauté française relative à l'organisation de l'éducation à la philosophie et à la citoyenneté en est un exemple paradigmatique. Elle a davantage été négociée entre le politique, le SeGEC et les syndicats des enseignants qu'elle n'a fait l'objet d'un débat au sein du Parlement communautaire ${ }^{86}$.

Concrètement, le résultat, s'il n'est pas exempt de critiques, n'est peut-être pas condamnable. Mais l'esprit cartésien, adepte d'une loi générale et abstraite - qui seule respecte le principe d'égalité -, n'y trouvera pas son compte.

Formellement en revanche, la législation organisant l'éducation à la philosophie et à la citoyenneté est inacceptable. Elle interdit en effet aux établissements libres confessionnels d'opter pour la création du nouveau cours, sans s'en justifier aucunement. L'on peut même considérer qu'elle méconnaît l'exigence de neutralité que lui assigne la Cour de Strasbourg lorsqu'elle se mêle d'enseignement. Pourquoi en effet ne pas avoir offert à ces établissements la liberté de choisir entre une approche transversale ou intégrée? Cette interdiction est particulièrement choquante pour les écoles d'une autre confession que le catholicisme - elles sont certes rares - qui ne sont pas représentées par le SeGEC. C'est aux établissements que la Constitution reconnaît la liberté d'enseignement, pas aux réseaux auxquels ils adhèrent. Même la liberté du réseau catholique est contrainte: s'il venait à changer d'option pédagogique et souhaitait abandonner la transversalité, il devrait quémander une modification législative.

À la faveur de l'institution du cours de philosophie et de citoyenneté, le législateur a été amené à moduler fortement l'interprétation de deux principes fondamentaux qu'il avait développés jusqu'alors, la liberté d'enseignement et sa neutralité.

La liberté d'enseignement constitutionnellement reconnue, notamment, aux établissements confessionnels, s'était érodée à mesure que croissait le souci d'assurer l'égalité des élèves par-delà les réseaux ${ }^{87}$. Elle recouvre ici une densité certaine, sous l'importante réserve qui vient d'être formulée qu'elle n'est pas accordée aux écoles mais aux réseaux. Au nom de cette

86. Dans sa proposition déposée le 7 décembre 2017 , le MR ne se risque pas à étendre le cours de philosophie et de citoyenneté à l'enseignement libre et se contente d'inviter le Gouvernement à prendre «langue avec le réseau libre subventionné afin de les convaincre d'organiser le cours de philosophie et citoyenneté dans les mêmes conditions que le fait le réseau officiel» (op. cit., p. 6).

87. V. M. El Berhoumi, Le régime juridique de la liberté d'enseignement à l'épreuve des politiques scolaires, op. cit., p. 435-524. 
liberté, le réseau libre confessionnel est dispensé de l'obligation de créer un cours spécifique de philosophie et de citoyenneté et se voit autorisé à assurer cette éducation de manière transversale. À l'heure où la Communauté française met en chantier une redéfinition des référentiels en toutes matières en vue d'améliorer les performances de son enseignement tant décrié par les enquêtes PISA ${ }^{88}$, il sera intéressant de voir si cette plus grande liberté sera de mise pour tous les programmes ou seulement pour la philosophie et la citoyenneté. Il est en effet malaisé de justifier pourquoi, au nom de la liberté confessionnelle de l'enseignement, une plus grande autonomie doit être reconnue pour cet enseignement que pour celui de la littérature et de l'histoire, par exemple, alors justement que l'éducation aux premières se fera dans le cadre du cours des secondes.

À l'instar de la République française, la Communauté française a développé une conception exclusive de la neutralité, qui l'a notamment conduite à encourager l'interdiction de signes convictionnels au sein de l'école ${ }^{89}$. Désormais, les professeurs qui combinent l'enseignement de la philosophie et la citoyenneté avec celui de la morale ou de la religion ne sont plus astreints, surtout dans le secondaire, qu'à une neutralité inclusive. L'on sait qu'en vertu de cette dernière, aussi appelée neutralité d'action, il est simplement imposé au prestataire du service public de traiter de façon égale et non discriminatoire les usagers du service public, tandis que selon la première, encore appelée neutralité d'apparence, l'agent ne doit pas seulement être neutre mais en avoir l'apparence, notamment en s'abstenant de toute expression convictionnelle ${ }^{90}$. Comment la Communauté française pourrait-elle justifier le maintien de sa conception exclusive de la neutralité

88. Socle de savoirs et de compétences du tronc commun. Charte des référentiels, doc. approuvé par le gouvernement le 25 oct. 2017: http://www.pactedexcellence.be [consulté le 3 janv. 2018]. V. B. D'Otreppe, «Le gouvernement fixe une méthode pour repenser les cours et leurs contenus», La Libre Belgique, 26 oct. 2017, p. 8-9.

89. V. H. Dumont et X. Delgrange, «Le principe de pluralisme face à la question du voile islamique en Belgique», art. cit., p. 75-108.

90. À propos de ces deux formes de neutralité, V. le Rapport final de la Commission du dialogue interculturel, précit., p. 54-56 et p. 115-119; S. VAn DrooghenBroeCK, «Les transformations du concept de neutralité de l'État: quelques réflexions provocatrices» et X. Delgrange, «Mixité sociale, mixité religieuse: le droit de l'enseignement face à la diversité », in J. RingelHeim (dir.), Le droit et la diversité culturelle, Bruxelles, Bruylant, 2011, p. 75 et p. 503, spéc. p. 76-79 et p. 533-534; V. DE COOREBYTER, «La neutralité n'est pas neutre», in D. Cabiaux et al. (dir.), Neutralité et faits religieux. Quelles interactions dans les services publics?, Louvain-la-Neuve, Academia-L'Harmattan, 2014, p. 19-43; J.-L. WolfS, «Former les enseignants à la "diversité" et à la "neutralité": est-ce conciliable? Analyse du cas de la Belgique francophone», in F. LORCERIE (dir.), Éducation et diversité. Les fondamentaux de l'action, Presses universitaires de Rennes, à paraître. 
à l'égard des élèves et des autres professeurs, s'agissant notamment de la question du port du voile?

\section{Remerciements}

Je remercie chaleureusement mes collègues du Conseil d'État de Belgique et de l'Université Saint-Louis - Bruxelles (au sein du CIRC, le Centre interdisciplinaire de recherches constitutionnelles), la Première auditrice Laurence Vancrayebeck et le Conseiller Luc Detroux, ainsi que Mathilde Philip-Gay, Professeure à l'Université Jean Moulin de Lyon et David Koussens, Professeur titulaire de la chaire de recherche sur les religions en modernité avancée de l'Université de Sherbrooke, pour les passionnantes discussions qui ont nourri l'écriture de ce texte.

Xavier Delgrange 